https://doi.org/10.22319/rmcp.v9i3.4194

Nota de investigación

\title{
Prevalencia de la acariosis traqueal y niveles de infestación de Acarapis woodi en colonias de abejas de Morelos, México
}

\section{Prevalence of honeybee tracheal mite disease and infestation levels of Acarapis woodi in honeybee colonies of Morelos, Mexico}

Claudia García Figueroa ${ }^{\mathrm{a}}$

Miguel Enrique Arechavaleta-Velasco ${ }^{\text {** }}$

${ }^{a}$ Centro Nacional de Investigación Disciplinaria en Fisiología y Mejoramiento Animal. Instituto Nacional de Investigaciones, Forestales, Agrícolas y Pecuarias. México.

*Autor de correspondencia: arechavaleta.miguel@inifap.gob.mx

\section{Resumen:}

Una de las principales enfermedades que afectan a las abejas melíferas es la acariosis traqueal causada por el ácaro Acarapis woodi. El objetivo de este estudio fue estimar la prevalencia de la acariosis traqueal y los niveles de infestación de Acarapis woodi en colonias de abejas del estado de Morelos, en donde la apicultura es un actividad pecuaria importante que se realiza bajo diversas condiciones climáticas y bajo diferentes sistemas de producción. El estudio se llevó a cabo utilizando muestras de abejas de 1,198 colonias que estaban ubicadas en 233 apiarios distribuidos a lo largo del territorio estatal, que son propiedad de 96 unidades de producción apícola. Los apiarios representan el $21.5 \%$ del total de apiarios que existen en el estado y las unidades de producción representan el $13.7 \%$ del total de unidades de producción registradas en Morelos. Los resultados indican que la prevalencia estimada para la acariosis traqueal en Morelos es de 0.02 y que el $10.30 \%$ de los apiarios incluidos en el estudio tuvieron al menos una colonia positiva a esta enfermedad. El porcentaje de infestación promedio en las colonias positivas fue de $7.32 \pm 0.75$, con un nivel mínimo de infestación de $5 \%$ y un nivel máximo de $20 \%$. Estos resultados indican que actualmente la acariosis traqueal tiene una prevalencia relativamente baja en Morelos y el porcentaje de infestación promedio estimado en las colonias positivas también es bajo, asimismo indican 
que la prevalencia estimada para esta enfermedad en este estudio es menor a la reportada hace 34 años.

- Palabras clave: Acariosis traqueal, Acarapis woodi, Abejas melíferas, Prevalencia.

\section{Abstract:}

Tracheal mite disease caused by Acarapis woodi is one of the most important diseases that affect honeybees. The objective of this study was to estimate the prevalence of tracheal mite disease and the levels of infestation of A.woodi in honeybee colonies in the State of Morelos, where beekeeping is an important activity performed in different climate conditions and under different production systems. This study was conducted using 1,198 honeybee colonies, located in 233 apiaries distributed along the state. The colonies belong to 96 beekeeping productive units. The apiaries included in the study represent $21.5 \%$ of the apiaries in the State and the beekeeping productive units represent the $13.7 \%$ of the beekeeping units registered in Morelos. The results of the study indicate that the prevalence of the tracheal mite disease in Morelos is 0.02 and that $10.30 \%$ of the apiaries included in the study had at least one colony infected with the disease. The average percent infestation in the positive colonies was $7.32 \pm 0.75$, with a minimum level of infestation of $5 \%$ and a maximum level of $20 \%$. These results indicate that currently the prevalence of the tracheal mite disease in Morelos is relatively low and that the average level of infestation estimated is low; the results indicate also that the prevalence estimated in this study for this disease is lower than the reported 34 yr ago.

Key words: Tracheal mites, Acarapis woodi, Honeybee, Prevalence.

Recibido 01/06/2016

Aceptado 25/09/2017

La apicultura es una actividad pecuaria importante y con mucho arraigo en Morelos, que se realiza en todo el estado bajo diversas condiciones climáticas ${ }^{(1)}$ y bajo diferentes sistemas de producción $^{(2)}$. El inventario apícola en la entidad es de 30,120 colmenas que se encuentran distribuidas en 1,084 apiarios. En 2014 se registró una producción de miel de 1,568t, lo que corresponde al $2.58 \%$ de la producción nacional ${ }^{(3)}$. 
En Morelos existen 700 unidades de producción apícola registradas que se clasifican de acuerdo a su objetivo y sistema de producción en: 1) productores de abejas reinas y núcleos, 2) productores de miel que movilizan colonias y 3) productores de miel que no movilizan colonias $^{(2,4)}$. Las abejas que manejan las unidades de producción son de origen europeo y africanizado $^{(5)}$. La actividad apícola en la entidad se realiza bajo tres tipos de clima: cálido subhúmedo $(\mathrm{A}(\mathrm{w}))$, semicálido $(\mathrm{ACw})$ y templado subhúmedo $(\mathrm{C}(\mathrm{w}))^{(1)}$.

Las abejas melíferas pueden sufrir el efecto de diversas enfermedades que afectan el desarrollo de las colonias y la producción de miel. En la mayoría de los casos, las pérdidas económicas pueden llegar a ser considerables, ya que los daños provocados van desde la reducción en la producción de miel hasta la pérdida total de la colonia.

Una de las principales enfermedades que afectan a las abejas melíferas es la acariosis traqueal $^{(6,7)}$. Esta enfermedad es una parasitosis interna causada por el ácaro Acarapis woodi, un parásito microscópico que vive en las tráqueas ubicadas en el tórax de las abejas, y fue descrito por primera vez por Rennie en $1921^{(6)}$.

Las abejas parasitadas pueden comportarse de manera normal; sin embargo, la población de abejas de una colonia se reduce cuando se encuentra infestada por $A$. woodi, lo que provoca que la producción de miel y la recolección de polen disminuyan ${ }^{(8,9)}$. Un estudio que se realizó en México, reporta que colonias con niveles de infestación considerados como moderados (35\%) produjeron en promedio $52 \%$ menos miel que colonias libres del parásito, mientras que colonias con niveles de infestación altos ( $89 \%$ ) produjeron en promedio $87 \%$ menos miel que colonias libres de este ácaro ${ }^{(10)}$.

La acariosis traqueal está presente en todos los países de importancia apícola del mundo con excepción de Australia, China y Japón. El primer reporte de esta enfermedad en México se registró en abril de 1980 en Jalisco, en apiarios cercanos a Guadalajara ${ }^{(11)}$. Estudios realizados durante 1982 reportaron la presencia de $A$. woodi en 24 de los 32 estados del país y se estimó una prevalencia a nivel nacional para la enfermedad de $30.5 \%{ }^{(12)}$.

Existen pocos estudios, publicados sobre la distribución de esta enfermedad en México. Un estudio que se realizó en Yucatán en 1992 reporta una prevalencia del $10 \%$ para la acariosis traqueal en la entidad ${ }^{(13)}$. Mientras, que otro estudio realizado en el 2009 en el municipio de Mérida en el que se incluyeron colonias de abejas silvestres y bajo manejo, reportó que no se detectó la presencia de $A$. woodi en las colonias incluidas en ese estudio ${ }^{(14)}$.

El único estudio publicado sobre la distribución de la acariosis traqueal en colonias de abejas de Morelos, se realizó en 1982, y se llevó a cabo con una muestra de 200 apiarios; se estimó que el $44.5 \%$ de los apiarios tuvieron colonias positivas a la enfermedad ${ }^{(12)}$. Con base en lo anterior, el objetivo de este estudio fue estimar la prevalencia de la acariosis traqueal y los niveles de infestación de A. woodi actuales en colonias de abejas del estado de Morelos. 
El estudio se llevó a cabo en 233 apiarios que fueron seleccionados en forma aleatoria; los apiarios incluidos en el estudio se encuentran distribuidos a lo largo del territorio estatal ${ }^{(15)}$, 115 apiarios estaban localizados en zonas con clima cálido subhúmedo $(\mathrm{A}(\mathrm{w})), 88$ apiarios en zonas con clima semicálido ( $\mathrm{ACw})$ y 30 en zonas con clima templado subhúmedo $(\mathrm{C}(\mathrm{w})$ ). Los 233 apiarios representan el $21.5 \%$ del total de apiarios que existen en el estado y pertenecen a 96 unidades de producción apícola, que representan el $13.7 \%$ del total de unidades de producción apícola registradas en Morelos. De los 233 apiarios incluidos en el estudio, 161 son propiedad de productores de miel que no movilizan colonias, 51 pertenecen a productores de miel que movilizan colonias y 21 a productores de abejas reinas y núcleos.

En cada uno de los apiarios se seleccionó en forma aleatoria al $20 \%$ de las colonias de abejas, seleccionándose en total 1,198 colonias. De cada colonia se obtuvo una muestra de aproximadamente 200 abejas obreras, que fueron recolectadas de uno de los bastidores centrales de la cámara de cría de las colmenas en frascos con alcohol al 70\%. Las muestras se tomaron entre los meses de diciembre de 2011 y marzo de 2012.

Para determinar el número de colonias infestadas y estimar el porcentaje de infestación de $A$. woodi de cada colonia, se utilizó el método descrito por Shimanuki y Knox ${ }^{(16)}$, para lo cual se tomaron 10 abejas de cada muestra y utilizando un microscopio estereoscópico se hicieron disecciones para separar el primer anillo torácico de las abejas para exponer las tráqueas. A cada anillo torácico se le aplicó una gota de ácido láctico al $85 \%$ para aclarar el tejido y separar los músculos torácicos de las tráqueas.

Veinticuatro horas después se observaron las tráqueas con ayuda de un microscopio óptico, las abejas que tuvieron tráqueas que presentaron manchas de color café-rojizo se identificaron como positivas ${ }^{(16)}$. Posteriormente, en las colonias que resultaron positivas se analizaron 10 abejas más, para determinar el número de abejas positivas en la muestra y estimar el porcentaje de infestación de la colonia, dividiendo el número de abejas positivas entre el número de abejas analizadas de la muestra $(n=20)^{(16)}$.

La prevalencia de la acariosis traqueal se calculó a partir de dividir el número de colonias positivas entre el total de colonias analizadas, mientras que el nivel de infestación promedio se estimó considerando el porcentaje de infestación de las colonias que fueron diagnosticadas como positivas a la enfermedad. Asimismo, se determinó el número de apiarios que presentaron al menos una colonia positiva a la enfermedad, para estimar la proporción de apiarios en los que la enfermedad está presente. Finalmente, para determinar si existe efecto del tipo de clima y del tipo de unidad de producción sobre la presencia de colonias positivas a la acariosis traqueal en los apiarios se realizaron pruebas de homogeneidad.

De las 1,198 colonias analizadas, únicamente 28 colonias resultaron positivas a la enfermedad. El porcentaje de infestación promedio en las colonias positivas fue de $7.32 \pm$ 0.75, con un nivel mínimo de infestación de $5 \%$ y un nivel máximo de $20 \%$. Las 28 colonias 
positivas estuvieron ubicadas en 24 apiarios de los 233 incluidos en el estudio. Con base en estos resultados la prevalencia estimada para la acariosis traqueal en Morelos es de 0.02 y el $10.30 \%$ de los apiarios incluidos en el estudio tuvieron al menos una colonia positiva a esta enfermedad.

Asimismo, se encontró que la presencia de colonias positivas a la enfermedad en los apiarios se distribuye en forma homogénea entre los tres tipos de climas en los que se realiza apicultura en la entidad $\left(X i^{2}=1.52 ; \mathrm{n}=233 ; P>0.05\right)$, y también se distribuye en forma homogénea entre los tres tipos de unidad de producción apícola que existen en Morelos $\left(X i^{2}=0.83 ; \mathrm{n}=233 ; P>0.05\right)$.

Estos resultados indican que actualmente la acariosis traqueal tiene una prevalencia relativamente baja en Morelos y el porcentaje de infestación promedio estimado en las colonias positivas también es bajo. Asimismo, muestran que no existe efecto del clima o del tipo de unidad de producción sobre la presencia de colonias positivas a la enfermedad en los apiarios.

Los resultados de este estudio indican que la prevalencia de esta enfermedad se redujo en forma importante durante los últimos 34 años, ya que en 1982, Zozaya et al ${ }^{(12)}$ reportaron que en Morelos la acariosis traqueal estaba presente en el $44.5 \%$ de los apiarios incluidos en su estudio $(n=200)$, mientras que en este trabajo se encontró que el $10.3 \%$ de los apiarios $(n=233)$ tuvieron al menos una colonia positiva. Diversos factores pudieron contribuir a la disminución que se observa; los factores más relevantes se discuten a continuación.

Después de que se detectó la enfermedad en 1982, inicialmente, los apicultores aplicaron productos químicos para controlarla, principalmente mentol y en menor medida bromopropylato (Folbex Forte ${ }^{\circledR}$ ) y una mezcla de nitrobenceno y salicilato de metilo (Acarol®); sin embargo, con el paso del tiempo los apicultores fueron dejando de aplicar productos químicos para controlar la enfermedad, a tal grado que se estima que no se han aplicado tratamientos contra este parásito en por lo menos los últimos 20 años. Actualmente los apicultores no realizan ninguna práctica de manejo o aplican algún producto químico para controlar esta enfermedad $^{(2)}$. Los productos químicos que se utilizaron en el pasado no eliminan completamente a los ácaros de una colonia ${ }^{(17)}$ y además es muy probable que en su momento, no todos los apicultores trataran sus colonias, de tal forma que es poco factible que la reducción en la prevalencia se deba a los tratamientos que se aplicaron en el pasado.

Algunos estudios que se realizaron en Yucatán y Veracruz, sugieren que las abejas africanizadas son más resistentes que las abejas europeas a la acariosis traqueal, pero no al grado de ser completamente resistentes al parásito ${ }^{(18,19,20)}$. Acarapis woodi se detectó en Morelos por primera vez en 1982, mientras que las abejas africanizadas arribaron a la entidad en $1990^{(21)}$; es posible que la disminución que se observa en la prevalencia esté relacionada con el proceso de africanización que ocurrió en la población de colonias de abejas, y a la 
interacción entre el parásito y las abejas africanizadas durante los últimos 26 años. Sin embargo, no todas las colonias de abejas en Morelos son africanizadas; en un estudio reciente se estimó que la frecuencia de colonias con morfotipo africanizado fue 0.28 , mientras que la frecuencia de las colonias europeas fue de 0.35 y la de las colonias con morfotipo híbrido o intermedio fue $0.37^{(5)}$, lo que indica que sólo una parte de las colonias de abejas que se manejan en la entidad son africanizadas, lo que indica que la africanización no puede explicar por sí sola la disminución en la prevalencia de $A$. woodi.

Se sabe que existe variabilidad genética en las poblaciones de colonias de abejas para la resistencia a la acariosis traqueal ${ }^{(22,23)}$, asimismo otro estudio demostró que es posible seleccionar colonias resistentes y susceptibles a este parásito ${ }^{(24)}$. La resistencia a este ácaro ha sido atribuida a la expresión del comportamiento de acicalamiento de las abejas ${ }^{(25,26,27)}$. La interacción que ha ocurrido entre las colonias de abejas y el parásito durante los últimos 34 años, pudo dar lugar a un proceso de selección natural en las poblaciones de abejas en respuesta a la presión de selección generada por la presencia de A. woodi. Es posible que la reducción que se observa en la prevalencia de la acariosis traqueal se deba en parte a este proceso.

La varroosis causada por Varroa destructor, es el principal problema desde el punto de vista sanitario que enfrenta la apicultura. Este ácaro se detectó en Morelos en 1993 y actualmente esta enfermedad tiene una alta prevalencia en el estado ${ }^{(28,29)}$. Los apicultores han utilizado productos químicos para el control de $V$. destructor, desde que el ácaro fue detectado en la entidad; los productos más utilizados son el fluvalinato, la flumetrina, el timol y el ácido oxálico $^{(2)}$. Existen reportes de que algunos de los productos utilizados para el control de $V$. destructor también actúan contra $A$. woodi. Se sabe que el fluvalinato ${ }^{(30)}$, el amitraz $^{(31,30,32)}$ y el timol ${ }^{(33,34)}$ también tienen efecto sobre $A$. woodi. Es probable que la disminución en la prevalencia de la acariosis se deba en parte a la constante aplicación de tratamientos contra $V$. destructor que se ha llevado a cabo durante los últimos 23 años, y que estos tratamientos hayan tenido un efecto indirecto sobre las poblaciones de $A$. woodi.

Se puede concluir que en la actualidad la prevalencia de la acariosis traqueal de las abejas en Morelos es baja y que los niveles de infestación promedio de Acarapis woodi en las colonias positivas también son bajos. Asimismo, los resultados indican que durante los últimos 34 años hubo una disminución importante en la prevalencia de esta enfermedad, a pesar de que en la actualidad los apicultores no aplican ningún tipo de control en contra de este parásito.

\section{- Literatura citada:}

1. Ornelas RF, Ambriz CR, Bustamante OJD. Delimitación y definición de agrohábitats en el estado de Morelos. Morelos, México: SAGARPA; 1997. 
2. Vélez IA, Espinosa JGA, Amaro GR, Arechavaleta VME. Tipología y caracterización de apicultores del estado de Morelos, México. Rev Mex Cienc Pecu 2016;7(4):507-524.

3. SIAP. Servicio de Información Agroalimentaria y Pesquera - Secretaría de Agricultura, Ganadería, Desarrollo Rural, Pesca y Alimentación. Disponible: http://www.siap.gob.mx/ganaderia-resumen-estatal-pecuario/. Consultado 6 Abr, 2016.

4. Vélez IA, Espinosa JGA, Arechavaleta VME, Vázquez PS. Caracterización tecnológica de apicultores del estado de Morelos. Congreso Nacional de Investigación Socioeconómica y Ambiental de la Producción Pecuaria. Toluca, Estado de México. 2013:189-198.

5. Arechavaleta VME, Vázquez PS, Ramírez RFJ, Camacho RC, Robles RCA, Amaro GR. Distribución de los morfotipos europeo, africanizado e híbrido en poblaciones de colonias de abejas de Morelos [resumen]. Reunión Nacional de Investigación Pecuaria. Veracruz, Ver. 2013:156.

6. Rennie J. Isle of Wight disease in hive bees - Acarine disease: The organism associated with the disease Tarsonemus woodi, n. sp. Transactions Royal Soc Edinburgh 1921;52:768-779.

7. Bailey L. Infectious diseases of the honey bee. England. Land Books; 1963.

8. Morse RA. Arachnids: Acarina (mites and ticks). En: Morse RA editor. Honey bee pests, predators and diseases. Ithaca, USA: Cornell University Press; 1978:197-209.

9. Denmark HA, Cromroy HL, Sanford MT. Honey bee tracheal mite, Acarapis woodi (Rennie) (Arachnida: Acari: Tarsonemidae). University of Florida, IFAS Extension. 2000.

10. Eischen FA, Cardoso-Tamez D, Wilson WT, Dietz A. Honey production of honey bee colonies infested with Acarapis woodi (Rennie). Apidologie 1989;20:1-8.

11. Wilson WT, Nunamaker RA. The infestation of honeybees in Mexico with Acarapis woodi. The American Bee J 1982;122(7):503-505.

12. Zozaya RJA, Guzmán NE, Tanus SE. Mexicans reports on acarine mite survey. The Speedy Bee 1982;10(12):16.

13. Echazarreta CM, García MA. Prevalencia de la acariosis traqueal en las abejas (Apis mellifera L. Hymenoptera: Apidae) [resumen]. Congreso Nacional de Entomología. San Luis Potosí, SLP. 1992:190-191. 
14. Martínez PJF, Medina MLA, Catzín VGA. Frecuencia de Varroa destructor, Nosema apis y Acarapis woodi en colonias manejadas y enjambres silvestres de abejas (Apis mellifera) en Mérida, Yucatán, México. Rev Mex Cienc Pecu 2011;2(1):25-38.

15. SEDAGRO. Secretaría de Desarrollo Agropecuario. Programa Morelos de Desarrollo Rural Sustentable. México. 2006.

16. Shimanuki H, Knox DA. Diagnosis of honey bee diseases. Agriculture Handbook. USA: U.S. Department of Agriculture; 2000.

17. Guzmán-Novoa E, Zozaya-Rubio A. The effects of chemotherapy on the level of infestation and production of honey in colonies of honey bees with acariosis. Am Bee $\mathrm{J}$ 1984;124(9):669-672.

18. Lagunas R, Vázquez M. Detección de acariosis traqueal por Acarapis woodi (Acarida) en enjambres de "abejas africanizadas" Apis mellifera scutellata y "abejas europeas" Apis mellifera ligustica en las cercanías de Nautla, Veracruz [resumen]. Congreso Nacional de Entomología. San Luis Potosí, SLP. 1992:192.

19. May-Itzá WDJ. Prevalencia de Nosema apis Z. y Acarapis woodi R. en colonias silvestres de abejas africanizadas y europeas (Apis mellifera L.) en el estado de Yucatán [tesis licenciatura]. Mérida, Yucatán: Universidad Autónoma de Yucatán; 1995.

20. Carrillo O. Prevalencia y distribución de Acarapis woodi (Rennie) y Nosema apis (Zander) en 15 sitios del estado de Yucatán en el año de 1992. Niveles de infestación y susceptibilidad en los ecotipos Africanizados y Europeas de Apis mellifera L. [tesis licenciatura]. Mérida, Yucatán: Universidad Autónoma de Yucatán; 1996.

21. Quezada-Euán JJG. A retrospective history of the expansion of Africanized honeybees in Mexico. J Apicultural Res 2007;46(4):295-300.

22. Gary NE, Page Jr. RE. Phenotypic variation in susceptibility of honey bees, Apis mellifera, to infestation by tracheal mites, Acarapis woodi. Exper Appl Acarol 1987;3(4):291-305.

23. Page Jr. RE, Gary NE. Genotypic variation in susceptibility of honey bees (Apis mellifera) to infestation by tracheal mites (Acarapis woodi). Exper Appl Acarol 1990;8(4):275-283.

24. Nasr ME, Otis GW, Scott-Dupree CD. Resistance to Acarapis woodi by honey bees (Hymenoptera: Apidae) - Divergent selection and evaluation of selection progress. J Econom Entomol 2001;94(2):332-338.

25. Pettis JS, Pakiw T. Grooming behavior by Apis mellifera L. in the presence of Acarapis woodi (Rennie) (Acari: Tarsonemidae). Apidologie 1998;29:241-253. 
26. Danka RG, Villa JD. Autogrooming by resistant honey bees challenged with individual tracheal mites. Apidologie 2003;34:591-596.

27. Danka RG, Villa JD. An association in honey bees between autogrooming and the presence of migrating tracheal mites. Apidologie 2005;36:331-333.

28. Vázquez PS, Arechavaleta VME, Ramírez RFJ, Amaro GR, Robles RCA. Prevalencia y niveles de infestación de Varroa destructor en colonias de abejas del estado de Morelos [resumen]. Reunión Nacional de Investigación Pecuaria. Veracruz, Ver. 2013:66.

29. García FC. Efecto del clima, el tipo de unidad de producción y el origen racial de las abejas sobre la varroosis en colonias de abejas en Morelos [tesis de licenciatura]. México, D.F. Universidad Nacional Autónoma de México; 2015.

30. Scott-Dupree CD, Otis GW. The efficacy of four miticides for the control of Acarapis woodi (Rennie) in a fall treatment program. Apidologie 1992;23(2):97-106.

31. Vandenberg JD, Shimanuki H. Effect of amitraz treatments on honey bees and on honey bee tracheal mite. Apidologie 1990;21:243-247.

32. Eischen FA, Vergara CH. Natural products smoke and its effect on Acarapis woodi and honey bees. Apidologie 2004;35:341-349.

33. Whittington R, Winston ML, Melathopoulos AP, Higo HA. Evaluation of the botanical oils neem, thymol, and canola sprayed to control Varroa jacobsoni Oud.(Acari: Varroidae) and Acarapis woodi (Acari: Tarsonemidae) in colonies of honey bees (Apis mellifera L., Hymenoptera: Apidae). Am Bee J 2000;140(7):567-572.

34. Calderone NW, Wilson WT, Spivak M. Plant extracts used for control of the parasitic mites Varroa jacobsoni (Acari: Varroidae) and Acarapis woodi (Acari: Tarsonemidae) in colonies of Apis mellifera (Hymenoptera: Apidae). J Econom Entomol 1997;90(5): 1080-1086. 